\title{
DISTINCTIVE TOMOGRAPHIC ABNORMALITIES OF THE CRANIOCERVICAL REGION IN A PATIENT WITH OSTEOGENSIS IMPERFECTA TYPE IV B
}

doi: $10.1590 / \mathrm{S1807-59322010000600014}$

\author{
Ali Al Kaissi, ${ }^{\mathrm{III}}$ Klaus Klaushofer, ${ }^{\mathrm{I}}$ Franz Grill ${ }^{\mathrm{II}}$
}

\section{INTRODUCTION}

Osteogenesis imperfecta is a clinically and genetically heterogeneous group of heritable disorders of connective tissue characterized by reduced bone mass (osteopenia) with associated bone fragility. The resulting skeletal manifestations are due to a generalized deficiency in the development of both membranous and endochondral bone and include markedly thin calvarium with delayed closure of the fontanelles and the sutures and excessive Wormian bone formation. Sillence et al. ${ }^{1}$ developed a classification system of OI subtypes: OI type I, which is characterised by blue sclerae; perinatal lethal OI type II, also known as congenital OI ; OI type III, a progressively deforming subtype with normal sclera; and OI type IV, which is characterized by a normal sclera. Levin et al. ${ }^{2}$ have suggested that OI subtypes could be further divided into type A and B based on the absence or presence of dentinogenesis imperfecta. Basilar impression involves the upward (vertical) migration of the odontoid process into the foramen magnum with a depression in the cranium. Basilar impression is a developmental defect and refers to the infolding of the occipital condyles, an elevation of the clivus, and the posterior cranial fossa of the skull. ${ }^{3}$ The soft bones of the skull base allow for progressive infolding of the dysplastic clivus and translocation of the odontoid into the posterior fossa. The combination of platybasia and basilar impression can lead to severe distortion of the spinal cord and the anterior brain stem. ${ }^{4-6}$ The specific structures that can be involved include the upper cervical cord, medulla, pons,

${ }^{\text {I }}$ Ludwig Boltzmann Institute of Osteology, Hanusch Hospital of WGKK and AUVA Trauma Centre Meidling, 4th Medical Department, Hanusch Hospital - Vienna, Austria.

II Orthopaedic Hospital of Speising, Paediatric Department - Vienna, Austria. Tel.: 004319102186924

Email: ali.alkaissi@osteologie.at mid-brain, cerebellum, as well as the vertebrobasilar system.

\section{CLINICAL REPORT}

Lateral skull radiographs showed significant frontal and occipital bossing, multiple wormian bones in the lambdoid suture associated with ill-defined skull base deformity and total loss of teeth (Figure 1). Anteroposterior skull radiograph showed excessive aeration of the frontal sinuses, osteopenia and total loss of teeth because of dentinogenesis imperfecta (Figure 2).

Strikingly, 3D sagittal CT scan showed assimilation of the entire anterior arch of the atlas with the foramen magnum. The odontoid process was greater than $7.8 \mathrm{~mm}$ above Chamberlain's line* (a) associated with thinned and dysplastic clivus. Note the Wachenheim-Clivus line** (b) in our patient the line does not intersect and is not tangential to the odontoid process (this confirms the existence of marked craniocervical abnormalities). Severe platybasia associated with significant distortion of the anatomical relations of the posterior fossa. Note the persistence of dentocentral synchondrosis (arrow) (Figure 3). An Axial reformatted CT scan revealed clefting of the anterior arch of the atlas and dysplasia of the posterior arch (arrows). The odontoid was impacted between the synchondrosis (Figure 4).

\section{DISCUSSION}

The modified Sillence system of classification differentiates OI into four types. Type I is marked by minor bone fragility and blue sclera, while type II is a lethal disorder. Type III consists of a group of sporadic cases that are mostly clinically and genetically heterogenous, and type IV has varying degrees of bone fragility with normal sclera. In general, OI type I is a purely qualitative collagen defect, whereas OI of type II, III, and IV are qualitative 


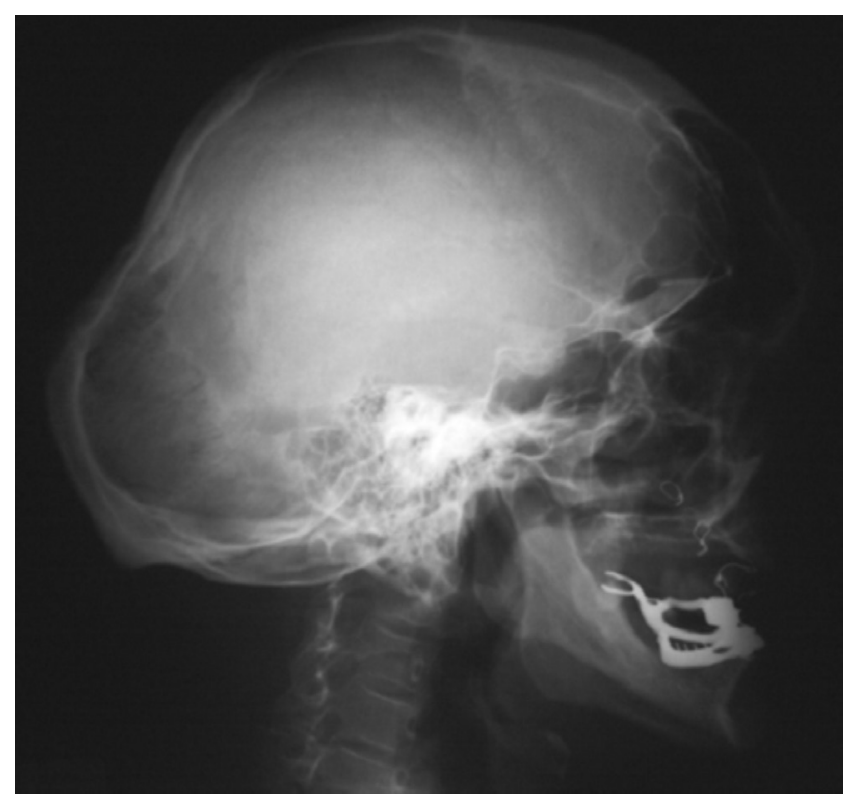

Figure 1 - Lateral skull radiographs showed significant frontal and occipital bossing, multiple wormian bones in the lambdoid suture associated with ill-defined skull base deformity and total loss of teeth.

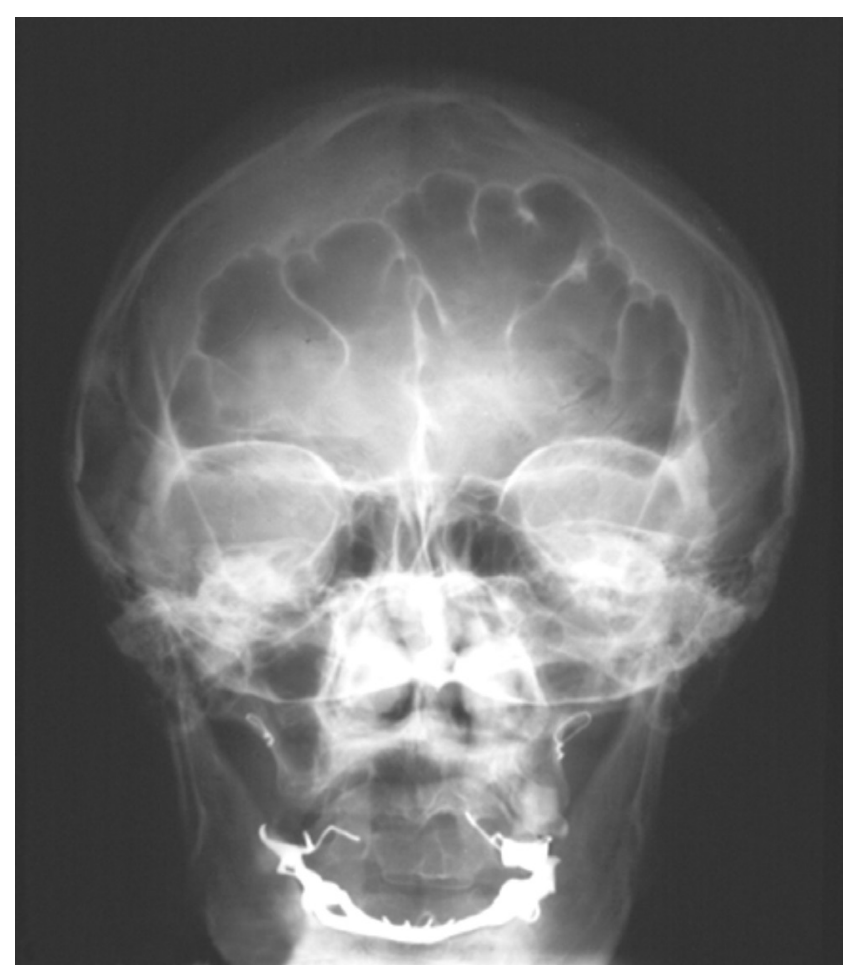

Figure 2 - Anteroposterior skull radiograph showed excessive aeration of the frontal sinuses, osteopenia and total loss of teeth because of dentinogenesis imperfecta.

and quantitative alterations in collagen synthesis. ${ }^{1-3}$ Levin et al. ${ }^{2}$ have proposed the addition of subtypes A and B to the modified Sillence classification system based on the absence or presence of dentinogenesis imperfecta, respectively. There have been a number of reports that

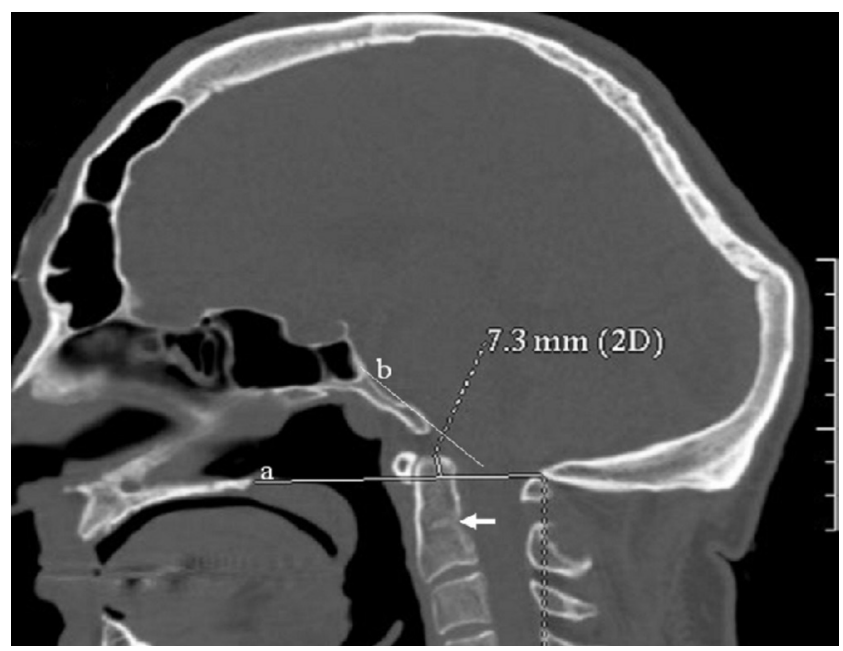

Figure 3 - A sagittal CT scan showed assimilation of the entire anterior arch of the atlas with the foramen magnum. The odontoid process was greater than $7.8 \mathrm{~mm}$ above Chamberlain's line* (a) associated with thinned and dysplastic clivus. Note the Wachenheim-Clivus line** (b) in our patient the line does not intersect and is not tangential to the odontoid process (this confirms the existence of marked craniocervical abnormalities). Severe platybasia associated with significant distortion of the anatomical relations of the posterior fossa. Note the persistence of dentocentral synchondrosis (arrow). At this stage we planned for transoral odontoidectomy and posterior fixation of the occiput with the spinous processes of $\mathrm{C} 2-\mathrm{C} 3 .{ }^{*}$ Chamberlain's line joins the hard palate to the posterior lip of the foramen magnum. Basilar impression is defined as being present when the tip of the dens projects more than $5 \mathrm{~mm}$ above Chamberlain's line. $3 * *$ Wachenheim clivus line (a method to evaluate and assess craniocervical junction abnormality/injury), a line drawn along the posterior aspect of the clivus toward the odontoid process. An abnormality is suspected when this line does not intersect and or / not tangential to the odontoid process. 8

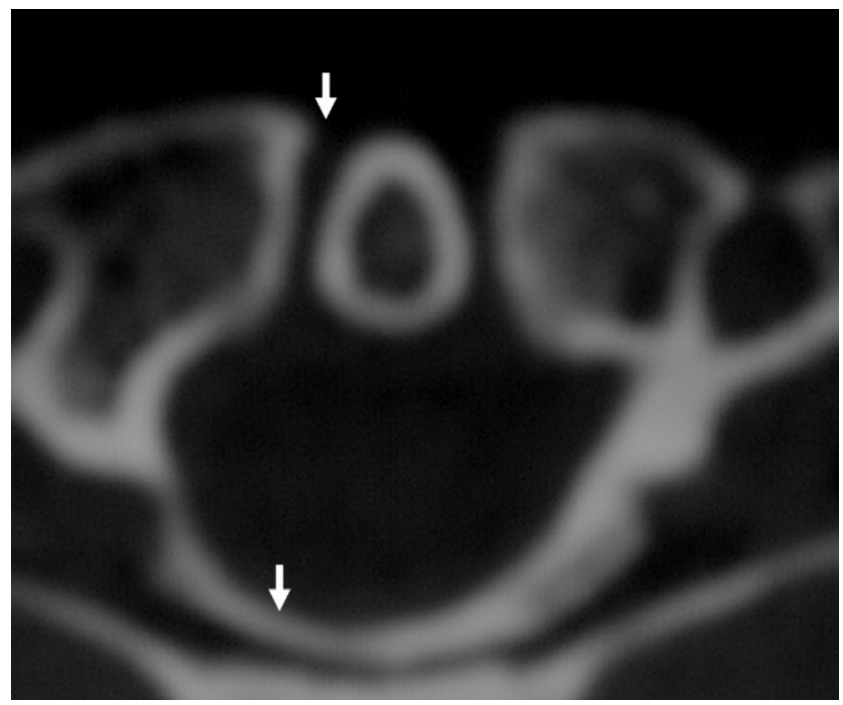

Figure 4 - An axial reformatted CT scan revealed clefting of the anterior arch of the atlas and dysplasia of the posterior arch (arrows). The odontoid has been impacted between the synchondrosis

have described the malformation complex in patients with OI type IVB. Engelbert et al. ${ }^{7}$ performed a questionnaire study to determine the prognosis of walking in patients with different types of OI. Interestingly, of 18 patients with 
OI type IV, 4 used wheelchairs. The mean age of the entire group was 11 years old. It should be noted, however, that basilar impression was neither screened for nor investigated in that study. Charnas and Marini ${ }^{9}$ reported their experience neuroimaging in 31 cases of OI type IV. Two of these cases were characterized by basilar invagination, three cases had macrocephaly, and two cases involved ventricular enlargement. Sillence ${ }^{4}$ recommended screening all patients with OI and particularly those with OI type IVB. He believes that basilar impression occurs with the highest frequency in patients with OI type IVB (patients with mild or moderate risk of fractures and normal scleras, but with dentinogenesis imperfecta). Most cases of basilar impression in patients with OI and or other forms of osteochondrodysplasias are probably due to the congenital bone malformation of the posterior fossa (primary basilar impression/invagination). ${ }^{10-13}$ MacKenzie et al. ${ }^{14}$ reported the sudden death of a patient with Marfan syndrome that was attributable to a primary type of basilar invagination. Secondary basilar impression, as seen in OI, results from abnormally soft bone that, in effect, slowly melts over the upright cervical spine, which progressively protrudes through the foramen magnum. ${ }^{3,4,5,12,13}$
Odontoid abnormalities have a reputation for being overlooked, resulting in a situation involving future instability and a potential risk for neurologic injury. Our present patient was characterized by a long odontoid/ basilar impression associated with the persistence of axial synchondrosis. The localization and level of the axial synchondrosis is not only important as an anatomical detail but it is also extremely important from a clinical perspective due to odontoid fractures..$^{15}$ The severe defective mineralization of the calvarium and its relevant osseous structures correlates directly with the development of basilar impression/ basilar invagination and can cause serious distortion of the brainstem. Finally, we need to stress that the sagittal reconstruction image of a multidetector $\mathrm{CT}$ scan is a useful investigative modality for assessing the extent of osseous malformation in asymptomatic patients with OI who are at a high risk of progression. MRI images, however, serve as a reference to predict possible neurological symptoms and signs. Therefore, the optimal imaging strategy for a particular patient will depend on the accurate clinico- radiographic assessment of the individual's underlying pathology.

\section{REFERENCES}

1. Sillence DO, Senn A, Danks DM. Genetic heterogeneity in osteogenesis imperfects. J Med Genet. 1979.16;110-6.

2. Levin LS, Salinas CF, Jorgenson RJ. Classification of dentinogenesis imperfecta by dental characteristics. Lancet.1978;1:332-3.

3. Chamberlain WE. Basilar impression/platybasia: Bizarre developmental anomaly of occipital bone and upper cervical spine with striking and misleading neurologic manifestations. Yale J Biol Med. 1939;11:478-96.

4. Sillence DO. Craniocervical abnormalities in osteogenesis imperfecta. Genetic and molecular correlation. Pediatr Radiol. 1994;24:427-30. Review

5. Hayes M, Parker G, Ell J and Sillence D: Basilar impression complicating osteogenesis imperfecta type IV: the clinical and neurological findings in four cases. J. Neurosurg. Psychiatry 1999;66:357-64.

6. Janus GJ, Engelbert RH, Beek E, Goosken RH, Pruijs JE. Osteogenesis imperfecta in childhood. MR imaging of basilar impression. Eur J Radiol.2003;47:19-24.

7. Engelbert RH, Uiterwaal CS, Gulmans VA, Pruijs H, Helders PJ. Osteogenesis imperfecta in childhood: prognosis for walking. J Pediatr. 2000;137:397-402.

8. Harris JH, Carson GC, Wagner LK. Radiologic diagnosis of traumatic occipitovertebral dissociation 2. Comparison of three methods. AJR. 1994;162:887-92.
9. Charnas LR, Marini JC. Communicating hydrocephalus, basilar invagination, and other neurologic features in osteogenesis imperfecta. Neurology. 1993;43:2603-8.

10. Al Kaissi A, Chehida FB, Ghachem M, Klaushofer K, Grill F. A hypoplastic atlas and long odontoid process in a girl manifesting phenotypic features resembling spondyloepimetaphyseal dysplasia joint laxity syndrome. Skeletal Radiol. 2008:37:469-73.

11. Al Kaissi A, Chehida FB, Gharbi H, Ghachem MB, Varga F, Klaushofer K. Persistent torticollis, facial asymmetry, grooved tongue, and dolichoodontoid process in connection with atlas malformation complex in three family subjects. Eur Spine J. 2007. Supp3:265-70.

12. Menzes AH. Specific entities affecting the craniocervical region: osteogenesis imperfecta and related osteochondrodysplasias: Medical and surgical management of basilar impression. Childs Nerv Syst. 2008:24:1169-72.

13. Ibrahim AG, Crockard HA. Basilar impression and osteogenesis imperfecta. J Neurosurg Spine. 2007;7:594-600.

14. MacKenzie JM, Rankin R. Sudden death due to atlantoaxial subluxation in Marfan syndrome. Am J Forensic Med Pathol. 2003;24:369-70.

15. Garton HJL, Park Papadopoulos SM. Fracture dislocation of the neurocentral synchondrosis of the axis. Case illustration. J Neurosurg Spine. 2002;96:350. 